\section{Medizin für Flüchtlinge: Öffnungsklausel nach §6 Asylbewerberleistungsgesetz mehr nutzen!}

\author{
Interview mit Oliver Razum und Judith Wenner
}
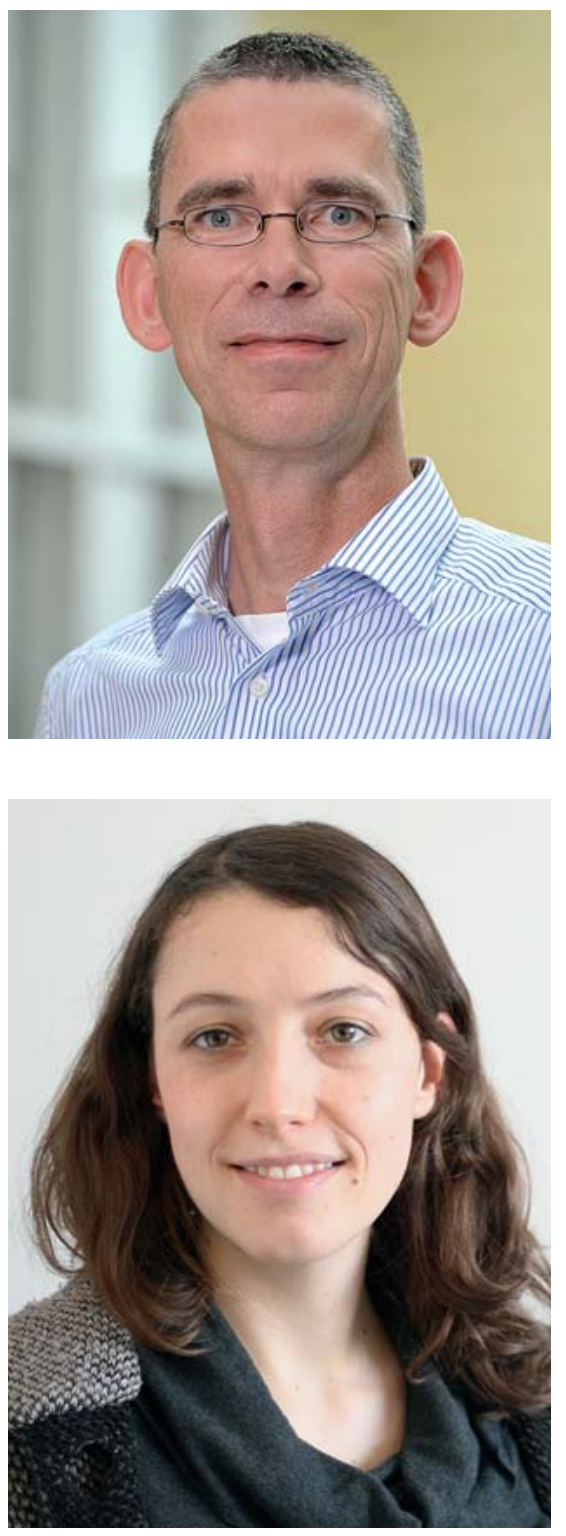

Die Gesundheitswissenschaftler Oliver Razum und Judith Wenner von der Universität Bielefeld plädieren dafür, dass Ärzte viel mehr von einer Öffnungsklausel im Asylbewerberleistungs-

1 Fortsetzung der Interviews zum Thema „Medizinische Versorgung den Flüchtlingen: teure Hürden“ (Z Orthop Unfall Heft 2/2017; 155: 129-135) gesetz Gebrauch machen. Und erklären, warum die eGK für Flüchtlinge im einwohnerstärksten Flächenland nur schleppend voran kommt.

Es gibt in NRW eine Vereinbarung des Landes mit 8 Kassen, die die elektronische Gesundheitskarte (eGK) für Asylbewerber verwalten würden, doch kommt die Einführung offenbar nur langsam voran, was ist los?

Wenner (W): In NRW ist es ähnlich wie in einer Reihe anderer Bundesländer so, dass es zwar einen Landesbeschluss gab. Dass der aber daran geknüpft wurde, dass auch noch mal in allen einzelnen Kommunen darüber entschieden wird. Im Prinzip würden Flüchtlinge die Karte dann erhalten, sobald sie nach spätestens 3 Monaten auf die Kommunen verteilt werden. Immer vorausgesetzt, die macht mit. Derzeit haben allerdings gerade mal 24 von 396 die eGK eingeführt. Darunter sind zumindest Köln und Düsseldorf mit vielen Einwohnern.

Razum (R): Das sind ja momentan noch nicht mal 10\% aller Kommunen. Und es sieht derzeit nicht so aus, als ob es rasch mehr werden. Etliche Kommunen fürchten offenbar höhere Kosten. Das bezieht sich nicht auf die tatsächlichen Kosten der Gesundheitsversorgung, sondern auf die höheren Verwaltungsausgaben, die von den Kassen erhoben werden. Ein Overhead von $8 \%$.

Und der ist vielen Kommunen zu teuer. W: Das ist zumindest der Eindruck, den auch wir haben, wobei das genau bei den Kommunen zu hinterfragen ist, um sich da nicht auf politische Spekulationen zu verlassen. Unser Ziel ist ja gerade, das zu hinterfragen und zu überprüfen. Dafür haben wir ein neues Projekt begonnen.

Welches Forschungsprojekt meinen Sie? W: Wir wollen im Projekt „Soziale Teilhabe durch Gesundheit: Analyse der Einführung einer elektronischen Gesundheitskarte für Geflüchtete in NRW“ in Kooperation mit
4 Kommunen untersuchen, welche Auswirkungen die Einführung der eGK auf alle Beteiligten hat. Und werden dafür sowohl mit den Geflüchteten sprechen, die die Karte benutzen können als auch mit Geflüchteten in anderen Kommunen, die keine Karte erhalten. Und auch mit anderen Beteiligten, etwa den Leistungserbringern und Vertretern der Kommunen.

\section{Es geht dabei vor allem um die Kosten?}

W: Längst nicht nur. Eine reine Kostenbetrachtung nützt nichts, wenn man sich nicht auch die Versorgung anschaut, die man zu welchen Kosten bekommt. Wir werden daher auch mit denen sprechen, die die Versorgung nutzen, welche Bewertung sie dazu finden, also die subjektive Seite erfragen. Qualitätsparameter kann man natürlich standardisieren. Dafür werden wir in Kooperation mit der AOK auch

\section{ZUR PERSON}

Prof. Dr. med. Oliver Razum (Jahrgang 1960) ist Leiter der AG Epidemiologie und International Public Health an der Fakultät für Gesundheitswissenschaft der Universität Bielefeld. Ein aktueller Arbeitsschwerpunkt ist Gesundheit und Migration womöglich auch die Spätfolge eines frühen Engagements in den Tropen. Von 1990 bis 1992 war Razum Medical Officer im Distrikt Chimanimani und Arzt am Chimanimani Hospital in Zimbabwe.

Judith Wenner (Jahrgang 1988) ist studierte Sozial- und Gesundheitswissenschaftlerin. Nach Auslandsaufenthalten in Ghana, Indien und Belgien arbeitet sie aktuell als wissenschaftliche Mitarbeiterin an der Fakultät für Gesundheitswissenschaft der Universität Bielefeld zum Kontext Flucht und Gesundheit. Wenner leitet aktuell 2 im Folgenden näher vorgestellte Projekte. 
die Inanspruchnahmen analysieren. Das Projekt fängt nächsten Monat an und wird 33 Monate dauern.

Prinzipiell ist die Versorgung aber doch geregelt. Auch die eGK würde in NRW nur den eingeschränkten Zugang nach Asylbewerberleistungsgesetz geben?

R: Das ist korrekt, auch in NRW gilt weiter §4 Asylbewerberleistungsgesetz. Allerdings, und das wird oft zu wenig beachtet, gilt auch $\S 6$, der es eigentlich erlaubt, die starren Einschränkungen im § 4 aufzuweichen. Es gibt dort ja die Möglichkeit für „sonstige Leistungen“ bei Bedarf im Einzelfall. Vorausgesetzt, der behandelnde Arzt oder die behandelnde Ärztin weiß das und nimmt das Ganze auch in Angriff.

Erkennt der Arzt an der Karte, dass ein Asylbewerber vor ihm steht?

W: Zunächst sieht man es nicht außen auf der Karte, aber es wird angezeigt, dass es sich um einen Asylbewerber handelt, sobald die Karte eingelesen ist.

Und jetzt ist es damit seine Aufgabe, die Regelungen im Asylbewerberleistungsgesetz, die $\S \S 4$ und 6 zu kennen, und dementsprechend die Leistungen einzuschränken? Ist es nicht so, dass vor allem die Sozialämter über solche Leistungen vorher entscheiden sollen?

R: Die Antwort ist in klares JEIN. Wenn ein Arzt oder Ärztin sich nicht an diese Einschränkungen hält, gleich verordnet, ohne vorher den Patienten beim Sozialamt nachfragen zu lassen, riskiert er oder sie Regressforderungen. Das ist das Risiko für ihn. Ob das wirklich greift, lässt sich nicht vorhersagen.

Auch eine Interpretationshilfe des Landes Sachsen vom Dezember 2015 hält fest, dass ein Diabetiker so behandelt werden muss, dass seine Krankheit sich nicht verschlechtert - Punkt 9: Prinzipiell müssen Diabetiker immer ausreichend eingestellt werden. In der Praxis, scheint es, wird das Gesetz nicht hart auf hart umgesetzt.

R: Lesen Sie mal weiter ...

Okay, hier... „Routineuntersuchungen beim Augenarzt und Facharztvorstellungen wegen Verdacht auf Folgeerkrankungen erfordern eine Kostenzusage...."
R: Man muss sich das mal auf der Zunge zergehen lassen, da ist ein Bundesland, das sagt seinen Ärzten, wenn jemand kommt, der dringend behandelt werden muss, dann müsst ihr behandeln. Andererseits aber muss eine womöglich dringend nötige Untersuchung beim Augenarzt erst bewilligt werden. Das kann doch nicht wahr sein. Es gibt ja so etwas wie ärztliche Therapiefreiheit. Die Ärztin oder der Arzt kann doch da nicht erst nachfragen.

Für uns sind die Begründungen dieser Leistungseinschränkung ohnehin in keinerlei Hinsicht nachvollziehbar.

\section{Warum nicht?}

R: Es gibt zum einen die Behauptung, es würden sehr hohe Kosten auf die Gemeinschaft zukommen, wenn man von Anfang an den Zugang in die Regelversorgung öffnet. Das halte ich aber für völlig unbewiesen, es spricht vieles für die Annahme, dass das Gegenteil der Fall sein dürfte. Nach den Analysen unserer Arbeitsgruppe zusammen mit Herrn Bozorgmehr von der Universität Heidelberg entstehen gerade durch die Einschränkungen der Leistungen höhere Kosten, als wenn man gleich den Zugang zur gesetzlichen Versicherung gewährt (siehe dazu das Interview Bozorgmehr ab S. 257). Das ist das ökonomische Argument.

Hinzu kommen Aspekte der Menschenrechte und der Menschenwürde. Wenn man Gesundheit als ein Menschenrecht akzeptiert, kann man nicht den Zugang zur Gesundheitsversorgung beschneiden. Und Deutschland hat eine Reihe von Konventionen dazu signiert und ratifiziert. Das geht zurück bis 1948, die Menschenrechtskonvention, bis zu aktuell EU-Richtlinien, die Deutschland mitgezeichnet hat, und die jetzt auf einmal selektiv abgewehrt werden.

Steht da nicht jetzt just das Land am Pranger, das seit 2015 eigentlich zumindest in Europa relativ viel für Flüchtlinge getan hat?

R: Ich sage nicht, dass Deutschland per se Flüchtlingen ein schlechtes Angebot macht. Aber zumindest bei der gesundheitlichen Versorgung schneidet Deutschland im sogenannten Migrant Integration and Policy Index (MIPEX) nicht gut ab.
Was ist das?

$R$ : Das ist ein fortlaufendes Vorhaben der International Organisation for Migration, in dem verschiedene Aspekte der Integration in Europa untersucht werden. Und da steht Deutschland, was Anspruch und Zugang zum Gesundheitswesen für Asylsuchende und für nicht dokumentierte Migranten angeht, unter den EU-Ländern nicht gut da: es kommt auf Platz 22 von 38. In der Summe über alle benutzten Parameter, die medizinische Versorgung ist bei diesem Ranking nur ein Aspekt, liegt Deutschland allerdings bei der letzten Auswertung von 2015 auf Platz 10 von insgesamt 38 Ländern [1].

Also lautet Ihre Forderung: Streichen wir die Einschränkungen gesundheitlicher Leistungen im Asylbewerberleistungsgesetz. Genau das, alias Zugang zur Kranken- und Pflegeversicherung ist allerdings im Oktober 2016 vom deutschen Bundestag abgelehnt worden. Politisch derzeit nicht durchsetzbar. Ein Gegenargument lautet etwa so, die Systeme sind voll, wir brauchen die Bremse des Asylbewerberleistungsgesetzes.

R: Die Frage ist, ob man eine Bremse aufrechterhält, die dazu führen kann, dass Menschen schwerer erkranken, und dann eventuell zu spät in den Gesundheitsdienst kommen. Herr Georg Classen von der Flüchtlingshilfe Berlin hat ausführlich Fälle dokumentiert, in denen wichtige medizinische Hilfe zu spät kam. Es sind gleichermaßen medizinische Tragödien, persönliche Tragödien wie auch eklatante Menschenrechtsverletzungen.

\section{Was tun?}

W: Solange sich politisch nichts ändert, bleibt die Frage, wie man §6 auslegt. Wenn ein Mediziner eine Behandlung für notwendig hält, dann wird sie über $\S 6$ abrechenbar. Wir fordern daher ausdrücklich eine stärkere Fokussierung auf §6. Man kann und sollte ihn nutzen.

R: Wenn alle wüssten, dass über § 6 eine Öffnungsklausel besteht, dann hätten wir ein viel kleineres Problem mit den Leistungseinschränkungen. Aber manche Mediziner wissen das auch nicht, das ist unsere Sorge. Und grundsätzlich stört uns eben, dass man dann, wenn man diesen Paragrafen anwenden muss, eigentlich 
konsequenterweise $\S 4$ und 6 ganz streichen sollte. Denn sonst entsteht eine Situation vollkommener Beliebigkeit.

Ein Problem scheint die Verordnung von Psychotherapie, die manche traumatisierten Flüchtlinge offenkundig benötigen.

W: Die Entscheidung darüber liegt primär bei den Kommunen. Wenn es einen akuten Behandlungsbedarf für Psychotherapie, dann gibt es eigentlich auch einen Anspruch. Und der wird auch durchaus gewährt.

Die Frage ist die praktische Realisierung in Form von therapeutischen Angeboten. Die in der jeweiligen Sprache sein müssen oder mit entsprechenden Dolmetschern. Wenn man aber faktisch keine Dolmetscher oder muttersprachlichen Behandler hat, ist es nicht die Frage des Anspruchs, sondern der praktischen Realisierung.

R: Das Thema wird seit vielen Jahren diskutiert. Und es betrifft nicht speziell nur
Asylsuchende, sondern generell Menschen mit Migrationshintergrund. Die Kassen sehen Dolmetscherleistungen nicht als Kassenleistung. Das ist seit langer Zeit ein strittiger Punkt.

Sie starten ein zweites Projekt zur Gesundheit von Flüchtlingen. Es geht um eine Vergleichsdatenbank für quantitative Forschung zu Gesundheit und gesundheitlicher Versorgung Geflüchteter. Was ist das?

W: Wir möchten da eine übersichtliche Datenbank aufbauen, um die Frage von Ungleichheit mit ein bisschen mehr Komplexität angehen zu können. Wir wollen nicht nur einfache Vergleiche machen zwischen Geflüchteten und Nichtgeflüchteten. Sondern auch den Einfluss anderer Merkmale wie Aufenthaltsdauer, Herkunftsländer beleuchten.

Im Sommer 2015 begann der große Zustrom von Flüchtlingen. Täuscht der Eindruck, dass die Forschung reichlich hin- tendran hängt? Sie bauen jetzt eine Datenbank auf, und wollen Fragen bearbeiten, zu denen wir längst Antworten bräuchten. Etwa dazu, welche gesundheitliche Versorgung brauchen denn die Migranten? R: Naja, wir zumindest arbeiten schon seit Jahren auf dem Gebiet, die bislang vermutlich einzige hiesige Studie zu den Kosten der Versorgung bei Asylbewerbern haben Herr Bozorgmehr und ich 2014 begonnen und 2015 publiziert. Was Migration insgesamt angeht, war glaube ich nicht voraussagbar, dass erst im Jahr 2015 das Ganze eskaliert. Dass es aber über kurz oder lang eskalieren würde, das war schon klar. Und sicherlich haben da manche Stellen ihre Hausaufgaben nicht gemacht.

\section{Literatur}

[1] Migration Immigration Policy Index (MIPEX) 2015: Key Findings. Im Internet: http://www. mipex.eu/key-findings; Stand: 13.02.2017

Das Interview führte Bernhard Epping. 